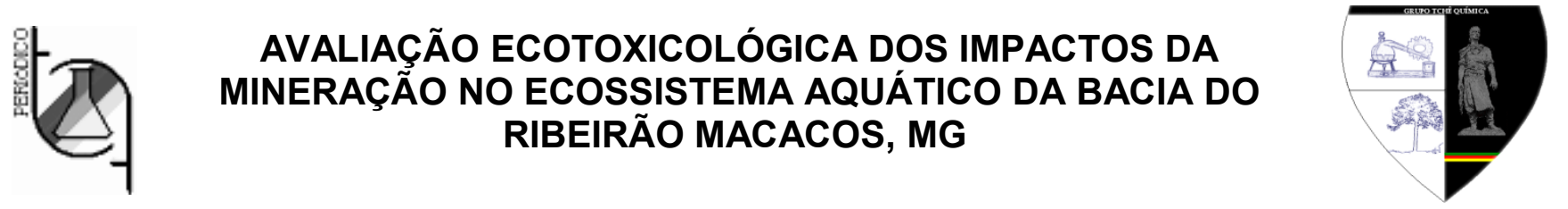

\title{
ECOTOXICOLOGICAL EVALUATION OF MINING IMPACTS ON AQUATIC ECOSYSTEM OF BASIN RIBEIRÃO MONKEYS, MG
}

\author{
MARIA, Marina A. ${ }^{1 *}$; SIUVES, Cláudia L. F. ${ }^{2}$; FERREIRA, Helena L. M. ${ }^{3}$; CASTRO, Paulo de T. A. ${ }^{4}$; \\ JUNIOR, Hermínio A. N. ${ }^{5}$ \\ 1,2,3 Instituto Senai de Tecnologia em Meio Ambiente Centro de Inovação e Tecnologia SENAI FIEMG - CITSF, \\ Avenida José Cândido da Silveira, 2000, Cep 31035-536, Belo Horizonte - MG, Brasil \\ (fone: +55 3134892000 fax: +55 313489 2200) \\ ${ }^{4,5}$ Departamento de Geologia, Escola de Minas, Universidade Federal de Ouro Preto, Rua Costa Sena, 171, \\ Cep 35400-000, Ouro Preto - MG, Brasil \\ (fone: +55 3135591602 fax: +55 313559 1600) \\ *Autor correspondente, e-mail: mamaria@fiemg.com.br
}

Received 10 February 2015; received in revised form 12 February 2015; accepted 04 May 2015

\begin{abstract}
RESUMO
A mineração, assim como outras alterações antrópicas podem gerar grandes impactos ambientais, alterando as características prístinas de diferentes biomas. Conhecer e compreender os seus potenciais efeitos auxilia na proposição de ações de controle e proteção dos recursos ambientais, em especial, dos ecossistemas aquáticos. O objetivo deste trabalho foi avaliar o efeito ecotoxicológico causado pelos impactos ambientais da mineração na bacia hidrográfica do ribeirão Macacos, no âmbito água e sedimento, somado à estudos físicoquímicos convencionais e quantificação de metais em diferentes sazonalidades avaliadas em duas coletas. A bacia apresentou graves indícios de degradação. Os parâmetros convencionais ainda não estão substancialmente alterados, assim como não apresenta ecotoxicidade aguda na água. No entanto o sedimento já se encontra com uma elevada concentração de diferentes metais, assim como efeitos ecotoxicológicos crônicos. A ocorrência desses eventos crônica, mesmo diante de resultados fisico-químicos normais, mostra a importância desses estudos tanto na água quanto no sedimento. As diferenças dos resultados obtidos nas duas campanhas demonstraram que a sazonalidade influenciou a disponibilidade da matéria orgânica do sedimento e/ou carreou por lixiviação.
\end{abstract}

Palavras-chave: Sedimento,água, ciclagem de contaminantes, efeito crônico, efeito agudo.

\section{ABSTRACT}

Mining, as well as other anthropogenic changes can have big environmental impacts by changing the pristine characteristics of different biomes. Know and understand their potential effects assists in proposing actions to control and protection of environmental resources, especially of aquatic ecosystems. The objective of this study was to evaluate the ecotoxicological effect caused by the environmental impacts of mining in the river watershed Monkeys, under water and sediment, added to the physical-chemical studies conventional and quantification of metals in different seasons evaluated in two collections. The basin had severe degradation of evidence. Conventional parameters are not substantially altered, and no acute ecotoxicity in water. However the pellet is already at a high concentration of various metals, as well as chronic ecotoxicological effects. The occurrence of these chronic events, even in the face of normal physical-chemical results, shows the importance of these studies both in the water and sediment. The differences in the results of the two campaigns showed that the seasonally influenced the availability of organic matter in the sediment and / or adduced by leaching.

Keywords: Sediment, water, cycling of contaminants, chronic effect, acute effect. 


\section{INTRODUÇÃO}

O presente estudo foi realizado na bacia do Ribeirão Macacos, município de Nova Lima, MG onde a mineração é a principal atividade econômica e está presente na região há mais de 40 anos. Esta atividade tem sido uma das principais responsáveis pelo assoreamento dos cursos de água que compõem a bacia. Porém, outros fatores de pressão ambiental também predominam, tais como esgotamento sanitário e erosões.

Este trabalho realizou a avaliação ecotoxicológica da água e sedimento de diferentes cursos d'água da bacia do Ribeirão Macacos, associada a caracterização físicoquímica convencional. A avaliação da ecotoxicidade em sedimento é importante por determinar se os compostos presentes nele podem ser prejudiciais aos organismos vivos tanto bentônicos, quanto os presentes na coluna d'água, uma vez que esses contaminantes podem ser liberados do sedimento $e$ resolubilizados a qualquer momento.

\section{PARTE EXPERIMENTAL}

\subsection{Caracterização da área de estudo}

A bacia do ribeirão Macacos possui uma área de $135,5 \mathrm{Km}^{2}$ e insere-se no município de Nova Lima. Suas nascentes surgem na Serra da Calçada e seus principais tributários da margem esquerda são: córrego dos Fechos (ou Fundo), Tamanduá, Taquaras, Gordura, Pai Coelho, do Moinho; e na margem direita tem-se: córrego Marumbé, Vieira, Pau de Rolha e Boiadeiros. A bacia do ribeirão Macacos encontra-se sobre rochas dos supergrupo Minas (em sua maior parte) e sobre o supergrupo Rio das Velhas. Suas cabeceiras drenam os quartzitos da Formação Moeda e os Itabiritos Formação Cauê [1].

A Bacia do Ribeirão Macacos está inserida entre áreas de preservação ambiental, como a APE de Fechos. No município de Nova Lima localiza-se a Área de Preservação Mata do Faria - com 76 ha. Os mananciais de água da bacia do Ribeirão Macacos abastecem Belo Horizonte. A vegetação da região é de transição de mata atlântica e cerrado, sendo a principal atividade a mineração de médio e grande porte e é também ocupada por condomínios residenciais e sítios.

\subsection{Estabelecimento da rede de amostragem}

Foram selecionados quinze sítios para realizar a caracterização da qualidade ecológica dos ambientes fluviais da bacia em estudo (Tabela 1). Esses sítios foram agrupados em sete bacias.

Foram realizadas duas campanhas de coleta. A primeira abrangeu o período de 27 de outubro a 28 de novembro de 2008 (transição entre o período seco e chuvoso) e a segunda de 4 a 31 de março de 2009 (período chuvoso).

\subsection{Caracterização das águas e sedimentos}

Em termos da qualidade físico-química das águas foram feitas medições instantâneas da temperatura, condutividade elétrica, $\mathrm{pH}$ e turbidez utilizando $\mathrm{O}$ aparelho portátil de medições com sonda multiparâmetro HORIBA U -10 .

Foram também coletadas amostras de água para execução dos seguintes ensaios e testes em laboratório: determinação da concentração do oxigênio dissolvido, demanda química de oxigênio, nitrogênio amoniacal, turbidez; teste de toxicidade aguda (organismo teste - Daphnia similis) e teste de toxicidade crônica (organismo teste Ceriodaphnia dubia).

As amostras foram coletadas a uma profundidade média de $20 \mathrm{~cm}$ da lâmina de água, preservadas e acondicionadas segundo as técnicas e métodos descritos em APHA (2005) [2]. Em laboratório, os ensaios foram realizados segundo APHA (2005) para os físico-químicos e segundo ABNT para ecotoxicológicos, sendo a NBR 12713 e 13373, respectivamente [3 e 4].

Os testes de toxicidade aguda foram feitos com diluição, podendo apresentar resultados quantitativos, expressos como CE50 (concentração efetiva mediana). Já os testes crônicos foram realizados sem diluição das amostras apresentando resultados qualitativos expressos como "Efeito crônico" ou "Não tóxico".

Amostras de águas e de sedimentos foram coletadas durante as campanhas com objetivo de analisar os metais presentes e a ecotoxicidade. Para quantificação de metais, foi utilizado o ICP-AES, analisadas nos laboratórios da UFOP/DEGEO. Para os ensaios ecotoxicológicos foi utilizado o solubilizado do sedimento, que é uma fração extraída do sedimento após a mistura de uma parte do 
sedimento à quatro partes de água de cultivo, submetida a $24 \mathrm{hs}$ de agitação, com posterior 12 hs de repouso para decantação, utilizando o sobrenadante para a realização dos ensaios.

\section{RESULTADOS E DISCUSSÃO:}

Com exceção do ponto MF02, da bacia do córrego Fechos, as análises físico-químicas convencionais não apresentaram nenhum valor extremo, sendo os demais condizentes com valores normais de águas superficiais. As alterações do ponto MF02 só ocorreram na primeira campanha, podendo ter ocorrido a coleta no momento de algum aporte de carga orgânica da ETE COPASA (Tabela 2).

A ecotoxicidade aguda não foi representativa uma vez que ocorreram apenas dois eventos com CE50 superior a $90 \%$, sendo um efeito muito baixo. Já os efeitos crônicos foram vários, tanto na água, quanto no sedimento, assim como nas duas coletas. Isso mostra a importância dos testes crônicos, pois muitas vezes os efeitos só podem ser percebidos após um período maior de exposição (tabela 3).

Os resultados dos testes de ecotoxicidade crônica representaram de forma fiel a atuação do sedimento como fonte e sumidouro de contaminantes. Pode-se observar que o efeito crônico só é observado em uma das tipologias amostrais, de forma que quando ocorre na água, não ocorre no sedimento e vice-versa, o que pode ser comprovado pela correlação inversa apresentada no teste estatístico de Spearman. Isso mostra que hora os contaminantes podem estar solubilizados na água e em outros momentos adsorvidos ou precipitados no sedimento.

Também é possível observar uma frequência muito maior de eventos crônicos no sedimento da primeira campanha, do que na segunda, o que pode ter ocorrido pelos eventos de chuva da segunda campanha, que deve ter aumentado a acidez da coluna d'água, aumentando a remoção de metais do sedimento, assim como a solubilização de carbonatos e hidróxidos, modificando o processo de dessorção de cátions metálicos complexados por matéria orgânica, devido à competição desses cátions com íons $\mathrm{H}+$. Esse processo pode ocorrer logo após as chuvas, que no geral são ácidas, reduzindo a ecotoxicidade do sedimento.
A quantificação de metais, só foi realizada no sedimento, de forma que não é possível avaliar a real distribuição dos mesmos na coluna d'água, mas foi importante por confirmar a presença de uma gama elevada de elementos, quase todos em concentrações bastante elevadas. A quantificação foi realizada nas amostras coletadas na segunda campanha, de forma que os valores poderiam ser ainda mais elevados na primeira campanha.

Não existe nenhuma legislação que trate de valores de referência para qualidade de sedimento, por isso utilizou-se para referência de alguns elementos, a resolução CONAMA/2004, que dá valores orientadores de metais em material a ser dragado em águas brasileiras. Os valores quantificados chegam a ser mais elevados que os valores permitidos para a disposição, estipulados na resolução [5].

A Tabela 4 apresenta valores da média de concentração para cada metal, na bacia do ribeirão Macacos como um todo, por isso foi acrescido dos valores de coeficiente de variação, para se ter uma noção da variação entre os pontos amostrados.

Foram realizadas análises estatísticas a fim de observar a ocorrência de diferenças entre as sete bacias monitoradas e entre as campanhas de amostragem. Como o número amostral foi relativamente baixo e alguns dos dados não apresentaram distribuição normal, optou-se pela utilização de estatística não paramétrica, que é a mais robusta para esses casos.

A análise estatística confirmou a não ocorrência de diferenças significativas entre as bacias para nenhum dos parâmetros na primeira, nem na segunda campanha. Ao juntar os dados das duas campanhas ouve diferença significativa para condutividade, que foi confirmada pelo teste de comparações múltiplas que identificou 0 córrego Fechos com valores significativamente maiores que o córrego Marumbé. Isso provavelmente devido a amostragem com valores elevados da primeira campanha de Fechos, sem maiores intervenientes.

Ao comparar as campanhas surgem mais algumas diferenças significativas, sendo os valores de OD e DQO significativamente menores na $1^{\mathrm{a}}$ campanha que na $2^{\mathrm{a}}$ campanha, enquanto que a ecotoxicidade crônica do 
sedimento foi significativamente maior na $1^{\text {a }}$ campanha. Esses resultados dizem muito, pois a tendência é que a chuva da segunda campanha eleve o OD e a DQO, por disponibilizar a matéria orgânica do sedimento, assim como o carreamento pela lixiviação. Assim como esses mesmos fatores levam a redução da toxicidade no sedimento (tabela 5).

Ainda foram realizados alguns testes de estatística multivariada para avaliar a similaridade entre os grupos e finalizar o raciocínio. A figura 1 mostra a similaridade entre as bacias através de um cluster e uma análise de componentes principais (ACP). Ambos ilustram a formação de três grupos, sendo um com os córregos Tamanduá, Mutuca e Gordura, outro com os córregos Boiadeiros, Marumbé e pouco afastado o ribeirão Macacos, e o terceiro seria o córrego Fechos, com a maior diferença entre todos. Se buscarmos na Tabela 1, podemos observar que o primeiro agrupamento citado, parece apresentar menos influência, tanto de mineração, quanto de carga orgânica, já o segundo, é o que apresenta maior número de pontos e todos com influência direta ou indireta de mineração e o córrego Fechos é a única bacia com a presença de ETE.

Para finalizar, também foi realizada uma ACP para a totalidade dos parâmetros monitorados, o que torna possível a visualização de conclusões já realizadas anteriormente, como a oposição da ecotoxicidade crônica para água e sedimento, assim como a aposição do OD ao grupo: DQO, turbidez, condutividade e nitrogênio amoniacal (Figura 2).

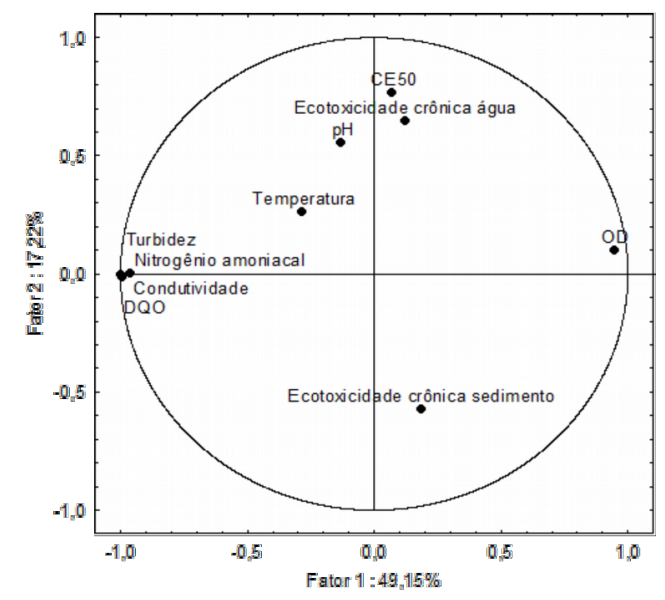

Figura 1. Análise de componentes principais dos parâmetros monitorados

\section{CONCLUSÕES:}

A bacia hidrográfica do ribeirão Macacos já apresenta graves indícios silenciosos de degradação. Os parâmetros convencionais ainda não estão substancialmente alterados, assim como não apresenta ecotoxicidade aguda na água, nem no sedimento, no entanto o sedimento já se encontra com uma elevada concentração de diferentes metais, assim como apresentou efeitos crônicos em diversos pontos e momentos do monitoramento.

As análises físico-químicas convencionais apresentaram valores condizentes com valores normais de águas superficiais, porém quase todos os pontos apresentaram eventos de ecotoxicidade. Este fato mostra a importância dos estudos ecotoxicológicos que evidenciam o comportamento e as transformações das substâncias químicas no meio ambiente, assim como os seus efeitos sobre os organismos que vivem nos ecossistemas aquáticos e terrestres. Os estudos ecotoxicológicos apresentam abordagens integradas dos efeitos de poluentes, levando em consideração a resposta da comunidade biológica.

É importante ressaltar a importância da realização de testes ecotoxicológicos em diferentes tipologias amostrais, como foi o caso da água e do sedimento que apresentaram respostas diferentes nas duas campanhas realizadas. Este fato pode estar relacionado à sazonalidade que pode influenciar a disponibilidade da matéria orgânica do sedimento e/ou carreamento por lixiviação.

Os resultados mostram que a ciclagem dos contaminantes ocorre constantemente acarretando diversas mudanças físico-químicas e diferentes formas de compartimentação de poluentes, o que permite que afete a organismos de todo o ecossistema aquático, sendo ele bentônico, nectônico ou planctônico. Lembrando que além dos efeitos tóxicos diretos causados aos organismos, pode haver também o acumulo e magnificação desses elementos nos organismos vivos e na cadeia alimentar, o que pode levar a consequências maiores, tanto de alteração da comunidade aquática, quanto da contaminação de seres humanos pela ingestão 
de alimentos, ou até mesmo de água contaminada por metais.

Sugere-se que trabalhos nessa linha, sejam complementados pela quantificação de metais também nas amostras de água, assim como realização de medidas físico-químicas in loco, também no sedimento, além de estudos de granulometria do sedimento e de acúmulo e biomagnificação em peixes.

\section{AGRADECIMENTOS:}

Agradecimentos à Universidade Federal de Ouro Preto (UFOP); à Fundação Centro Tecnológico de Minas Gerais (CETEC); à Fundação de Amparo a Pesquisa do Estado de Minas Gerais (FAPEMIG).

\section{REFERÊNCIAS:}

1. ASSOCIAÇÃO BRASILEIRA DE NORMAS TÉCNICAS - ABNT. NBR 12713: Ecotoxicologia aquática -
Toxicidade aguda - Método de ensaio com Daphnia spp (Crustacea, Cladocera). Rio de Janeiro, 2006.

2. ASSOCIAÇÃO BRASILEIRA DE NORMAS TÉCNICAS - ABNT. NBR 13373: Ecotoxicologia aquática Toxicidade crônica - Método de ensaio com Ceriodaphnia spp (Crustacea, Cladocera). Rio de Janeiro, 2006. 15 p.

3. CONSELHO NACIONAL DE MEIO AMBIENTE $n^{\circ}$ 344. Estabelece as diretrizes gerais $\mathrm{e}$ os procedimentos mínimos para a avaliação do material a ser dragado em águas jurisdicionais brasileiras, e dá outras providências, de 25 de março de 2004.

4. DOOR II, J.V.N. Phisiographic, stratigraphic and structural development of the. Quadrilátero Ferrífero, Minas Gerais, Brazil. Washington: DNPM/USGS, 1969.

5. STANDARD methods for the examination of water and wastewater (SM) 21 ed. Washington: APHA, AWWA, WEF, 2005. $1 \mathrm{v}$.

Tabela 1. Resultados estatísticos

\begin{tabular}{c|c|c|c|c}
\hline \multirow{2}{*}{ Parâmetros } & \multicolumn{3}{|c|}{ Kruskal-wallis “p” } & Entrecampanhas \\
\cline { 2 - 5 } & $\begin{array}{c}\text { Entrebacias } \\
\mathbf{1}^{\mathbf{a}} \text { campanha }\end{array}$ & $\begin{array}{c}\text { Entrebacias } \\
\mathbf{2}^{\mathbf{a}} \text { campanha }\end{array}$ & $\begin{array}{c}\text { Entrebacias } \\
\text { Geral }\end{array}$ & Mann-Whitney “p” \\
\hline OD & 0,42 & 0,74 & 0,58 & 0,01 \\
\hline $\begin{array}{c}\text { Ecotoxicidade } \\
\text { crônica água }\end{array}$ & 0,49 & 0,54 & 0,74 & 0,02 \\
\hline $\begin{array}{c}\text { Nitrogênio } \\
\text { Amoniacal }\end{array}$ & 0,43 & 0,12 & 0,20 & 1,00 \\
\hline $\begin{array}{c}\text { Turbidez } \\
\text { Temperatura da } \\
\text { água }\end{array}$ & 0,85 & 0,30 & 0,44 & 0,38 \\
\hline $\begin{array}{c}\text { Condutividade } \\
\text { elétrica }\end{array}$ & 0,55 & 0,43 & 0,20 & 0,68 \\
\hline pH & 0,18 & 0,24 & 0,19 & 0,16 \\
\hline $\begin{array}{c}\text { Ecotoxicidade } \\
\text { crônica sedimento }\end{array}$ & 1,00 & 0,24 & 0,00 & 0,57 \\
\hline
\end{tabular}

PERIÓDICO TCHÊ QUÍMICA • www.periodico.tchequimica.com • Vol. 12 N. 24

• ISSN 1806-0374 (impresso) • ISSN 1806-9827 (CD-ROM) • ISSN 2179-0302 (meio eletrônico)

(C) 2015. Porto Alegre, RS. Brasil

The Periódico Tchê Química (ISSN: 1806-0374; 2179-0302) is an open-access journal since 2004. Journal DOI: 10.52571/PTQ. http://www.tchequimica.com This text was introduced in this file in 2021 for compliance reasons.

OPEN ACCESS. This article is licensed under a Creative Commons Attribution 4.0 (CC BY 4.0) International License, which permits use, sharing, adaptation, distribution, and reproduction in any medium or format, as long as you give appropriate credit to the original author(s) and the source, provide a link to the Creative Commons license, and indicate if changes were made. The images or other third-party material in this article are included in the or exceeds the permilted use you will need to oblin peris or exceeds the permitted use, you will need to obtain permission directly from the copyright holder. To view a copy of this license, visit http://creativecommons.org/licenses/by/4.0/. 